\title{
Quantum chemical studies on acidity-basicity behaviors of some bipyridine derivatives
}

\author{
C.Öğretir, '̇. İ. Öztürk, N. F. Tay \\ Eskişehir Osmangazi University, Faculty of Arts \& Sciences, Chemistry Department, 26040 \\ Eskişehir, Turkey \\ E-mail: cogretir@ogu.edu.tr
}

\begin{abstract}
The protonation of six isomeric forms of bipyridine has been investigated by means of different quantum chemical methods (i.e. semi-empirical (PM5), DFT (B3LYP/6-31G(d)) and ab initio $(\mathrm{HF} / 6-31 \mathrm{G}(\mathrm{d}))$. It is concluded that 2,2'-bipyridine exists as transoid isomer, 2,3'-bipyridine as cisoid isomer and 3,3'-bipyridine coexist in equal amount of cisoid and transoid isomeric forms.
\end{abstract}

Keywords: Bipyridines, acidity constants, nucleophlicity, proton affinities, substituent constant, isomers

\section{Introduction}

Many procedures were reported for the synthesis of bipyridine derivatives in the past years. It seems that the substituted bipyridine derivatives have been prepared mostly by reactions of Grignard reagents or organo-lithium derivatives with various sulfur and phosphor containing compounds respectively. ${ }^{1-9}$

Studies on structure of bipyridine derivatives are also indicate an enormous amount of research affords. A low temperature X-ray structure, ${ }^{10}$ the theoretical calculations ${ }^{11-15}$ and spectroscopic studies of bipyridine derivatives have been reported in the literature. ${ }^{16}$

Chemical reaction studies of the various bipyridines are numerous. Among the other isomers the chemistry of the 2,2'-bipyridine is the most studied because of its ability to complex with metals. ${ }^{17-21}$ This compound usually acts as a chelating ligand. ${ }^{22-26}$

Much of the chemistry carried out on the bipyridines has been for their incorporation as structural motifs in large molecules and supramolecular species. ${ }^{27-33}$

Following our previous work on alkyl and halogen substituted pyridines ${ }^{34-39}$ and bipyrazoles, ${ }^{40}$ we now are reporting on the semi empirical (PM5) and ab initio (HF and DFT) calculated structural and thermodynamic parameters of six isomeric bipyridine derivatives (Scheme 1). Any possible parallelism with reported and available experimental data were searched. The parallelism and discrepancies between the experimental and computed data were 
attempted to be interpreted.

\section{Computational methods}

Theoretical calculations were carried out at the restricted Hartre-Fock level (RHF) using PM5, method in the MOPAC 2002 program ${ }^{41 a}$ implemented in an Intel Pentium IV computer. All the structures were optimized to a gradient norm of and $0.1-1.0$ in the aqueous phase, using the eigenvector method following (EF).The initial estimates of the geometry of all the structures were obtained by a molecular mechanics program of CS Chem. Office pro for Windows ${ }^{42}$, followed by full optimization of all geometrical variables (bond lengths, bond angles and dihedral angles), without any symmetry constraint, using the semi-empirical PM5 quantum chemical methods in the MOPAC 2002 program.

Nowadays both microscopic and macroscopic theoretical methods are available for the estimation of solvation free energies. Therefore it is possible, in principle, to determine theoretical relative or absolute acidity constants, $\mathrm{pK}_{\mathrm{a}}$ values, the thermodynamic cycle using the Equation 1.

Scheme 1 explains the interrelationship between the thermodynamic parameters of gas and solution phases.

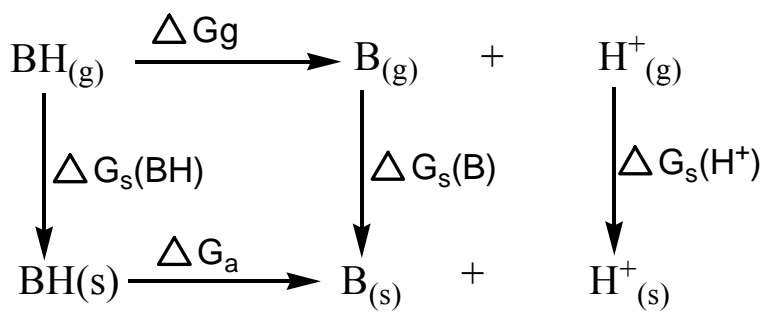

Scheme 1. Interrelationship between the gas phase and solution thermodynamic parameters.

The acidity constant, $\mathrm{pK}_{\mathrm{a}}$, can be computed by using Equation 1 and Equation 2. The Equation 2 is an rearranged form of Equation 1.

$$
\begin{aligned}
& \Delta \mathrm{G}=-\mathrm{RT} \ln \mathrm{K}_{\mathrm{a}} \\
& \mathrm{pK}_{\mathrm{a}}=\Delta \mathrm{G}_{\mathrm{a}} / 2.303 \mathrm{RT}
\end{aligned}
$$

The $a b$ initio calculation of the absolute $\mathrm{pK}_{\mathrm{a}}$ values can be made by using Equation 3 in which $\Delta \mathrm{G}_{\mathrm{g}}$ and $\Delta \mathrm{G}_{\mathrm{a}}=\Delta \mathrm{G}_{\mathrm{s}}(\mathrm{B})-\Delta \mathrm{G}_{\mathrm{s}}(\mathrm{BH})+\Delta \mathrm{G}_{\mathrm{s}}\left(\mathrm{H}^{+}\right)$are the gas phase and solvation free energies of the ionization and $\Delta \mathrm{G}_{\mathrm{s}}$ 's are solvation free energies respectively. ${ }^{43,44}$

$$
\mathrm{pK}_{\mathrm{a}}=\left[\Delta \mathrm{G}_{\mathrm{g}}+\Delta \mathrm{G}_{\mathrm{a}}\right] / 2.303 \mathrm{RT}
$$


In the present work ab initio Hartree-Fock (HF) and density functional geometry (DFT) optimizations were performed using the Gaussian 03 program. ${ }^{41 \mathrm{~b}}$ The calculation of solvation free energies was carried out using at $\mathrm{HF} / 6-31 \mathrm{G}(\mathrm{d})$ and $\mathrm{B} 3 \mathrm{LYP} / 6-31 \mathrm{G}(\mathrm{d})$ respectively. The value of $\Delta \mathrm{G}_{\mathrm{s}}\left(\mathrm{H}^{+}\right)-271.2 \mathrm{kcal} \mathrm{mol}^{-1}$ was used as suggested in the literature. ${ }^{44}$

\section{Result and Discussion}

The computed physical and thermodynamic parameters for studied compounds were depicted in Tables 1-3. We have attempted to evaluate the obtained results as follows:

\section{Acidity- basicity}

The acidity of a given base, B, for the protonation reaction (Equation 4) can be calculated using the Equation 5 , where $\Delta \mathrm{G}$ is the standard free energy. ${ }^{45}$

$$
\begin{aligned}
& \mathrm{B}:+\mathrm{AH}^{+}=\mathrm{BH}^{+}+\mathrm{A} \\
& \delta \Delta \mathrm{G}_{(\mathrm{BH}+)}=\left[\Delta \mathrm{G}_{(\mathrm{B})}+\Delta \mathrm{G}_{(\mathrm{AH}+)}\right]-\left[\Delta \mathrm{G}_{(\mathrm{BH}+)}+\Delta \mathrm{G}_{(\mathrm{A})}\right]
\end{aligned}
$$

In Equation 1, $\mathrm{B}$ and $\mathrm{BH}^{+}$are neutral and protonated species of base $\mathrm{B}$, and $\mathrm{A}$ and $\mathrm{AH}^{+}$are $\mathrm{H}_{2} \mathrm{O}$ and $\mathrm{H}_{3} \mathrm{O}^{+}$respectively. The computed thermodynamic data were used in predicting the $\mathrm{pK}_{\mathrm{a}}$ values of various species, using Equation 6 in which the $\delta \Delta \mathrm{G}_{(\mathrm{BH}+)}$ is the standard free energy change for protonation reaction (Equation 4, Table 4 and 5).

$$
\mathrm{pK}_{\mathrm{a}(\mathrm{BH}+)}=\delta \Delta \mathrm{G}_{(\mathrm{BH}+)} / 2.303 \mathrm{RT}
$$

\begin{tabular}{|c|c|c|c|c|c|c|c|}
\hline $\begin{array}{c}\text { Compo } \\
\text { und }\end{array}$ & $\begin{array}{c}\Delta \mathrm{H}_{f} \\
(\mathrm{kcal} / \mathrm{mol})\end{array}$ & $\begin{array}{c}\Delta \mathrm{S} \\
(\mathrm{cal} / \mathrm{molK})\end{array}$ & $\begin{array}{c}\Delta \mathrm{G}_{f} \\
(\mathrm{kcal} / \mathrm{mol})^{\mathrm{a}}\end{array}$ & $\begin{array}{c}\text { Compo } \\
\text { und }\end{array}$ & $\begin{array}{c}\Delta \mathrm{H}_{f} \\
(\mathrm{kcal} / \mathrm{mol})\end{array}$ & $\begin{array}{c}\Delta \mathrm{S} \\
(\mathrm{cal} / \mathrm{molK})\end{array}$ & $\begin{array}{c}\Delta \mathrm{G}_{f} \\
(\mathrm{kcal} / \mathrm{mol})^{\mathrm{a}}\end{array}$ \\
\hline $1 \mathrm{~s}$ & 51.77 & 88.55 & 25.38 & $1 \mathrm{t}$ & 51.67 & 88.39 & 25.33 \\
\hline 1sN'p & 153.33 & 90.40 & 126.39 & 1tN'p & 155.15 & 90.60 & 128.15 \\
\hline $1 \mathrm{sNp}$ & 153.29 & 90.37 & 126.36 & $1 \mathrm{tNp}$ & 155.14 & 90.33 & 128.22 \\
\hline $1 \mathrm{sN}$ '’p & 260.144 & 95.79 & 231.59 & $1 \mathrm{tN}$ 'p & 263.05 & 89.78 & 236.30 \\
\hline $2 s$ & 50.19 & 89.80 & 23.43 & $2 t$ & 50.37 & 89.87 & 23.59 \\
\hline $2 s N^{\prime} p$ & 151.57 & 90.56 & 124.58 & 2tN'p & 151.84 & 90.32 & 124.92 \\
\hline $2 \mathrm{sNp}$ & 153.71 & 90.89 & 126.62 & $2 \mathrm{tNp}$ & 153.43 & 90.60 & 126.43 \\
\hline 2sN"'p & 257.57 & 90.82 & 230.50 & $2 \mathrm{tN}$ "p & 257.60 & 90.80 & 230.54 \\
\hline
\end{tabular}

Table 1. Aqueous phase PM5 calculated thermodynamic data for studied molecules for cisoid and transoid forms $(\varepsilon: 78.4)$ 
Table 1. Continued

\begin{tabular}{cccccccc}
\hline $\begin{array}{c}\text { Compo } \\
\text { und }\end{array}$ & $\begin{array}{c}\Delta \mathrm{H}_{\mathrm{f}} \\
(\mathrm{kcal} / \mathrm{mol})\end{array}$ & $\begin{array}{c}\Delta \mathrm{S} \\
(\mathrm{cal} / \mathrm{molK})\end{array}$ & $\begin{array}{c}\Delta \mathrm{G}_{\mathrm{f}} \\
(\mathrm{kcal} / \mathrm{mol})^{\mathrm{a}}\end{array}$ & $\begin{array}{c}\text { Compo } \\
\text { und }\end{array}$ & $\begin{array}{c}\Delta \mathrm{H}_{\mathrm{f}} \\
(\mathrm{kcal} / \mathrm{mol})\end{array}$ & $\begin{array}{c}\Delta \mathrm{S} \\
(\mathrm{cal} / \mathrm{molK})\end{array}$ & $\begin{array}{c}\Delta \mathrm{G}_{\mathrm{f}} \\
(\mathrm{kcal} / \mathrm{mol})^{\mathrm{a}}\end{array}$ \\
\hline $\mathbf{3}$ & 50.81 & 90.05 & 23.98 & & & & \\
$\mathbf{3 N ' p}$ & 151.23 & 90.41 & 124.29 & & & & \\
$\mathbf{3 N p}$ & 154.49 & 90.43 & 127.54 & & & & \\
$\mathbf{3 N}$ 'p & 253.24 & 96.32 & 224.53 & & & & \\
$\mathbf{4 s}$ & 49.78 & 88.68 & 23.35 & $\mathbf{4 t}$ & 49.79 & 88.70 & 23.36 \\
$\mathbf{4 s N ' p}$ & 150.92 & 90.35 & 124.00 & $\mathbf{4 t N ' p}$ & 150.90 & 90.36 & 123.97 \\
$\mathbf{4 s N p}$ & 151.00 & 90.27 & 124.10 & $\mathbf{4 t N p}$ & 150.91 & 90.34 & 123.99 \\
$\mathbf{4 s N}$ 'p & 253.55 & 89.47 & 226.88 & $\mathbf{4 t N}$ 'p & 253.54 & 90.08 & 226.69 \\
$\mathbf{5}$ & 49.64 & 89.97 & 22.83 & & & & \\
$\mathbf{5 N ' p}$ & 149.68 & 90.55 & 122.70 & & & & \\
$\mathbf{5 N p}$ & 151.30 & 90.45 & 124.35 & & & & \\
$\mathbf{5 N}$ 'p & 252.70 & 90.85 & 225.62 & & & & \\
$\mathbf{6}$ & 50.07 & 87.26 & 24.07 & & & & \\
$\mathbf{6 N ' p}$ & 150.57 & 89.15 & 124.00 & & & & \\
$\mathbf{6 N p}$ & 150.59 & 89.17 & 124.02 & & & & \\
$\mathbf{6 N}$ 'p & 252.30 & 88.29 & 225.99 & & & & \\
\hline
\end{tabular}

${ }^{\mathrm{a}}$ from $\Delta G_{f}=\Delta \mathrm{H}_{f}-\mathrm{T} . \Delta \mathrm{S}$

Pyridine (PM5) : $\Delta \mathrm{H}_{\mathrm{f}}(\mathrm{kcal} / \mathrm{mol})=22.99, \Delta \mathrm{S}(\mathrm{cal} / \mathrm{molK})=68.10, \Delta \mathrm{G}_{\mathrm{f}}(\mathrm{kcal} / \mathrm{mol})=2.70$.

Pyridinium (PM5) : $\Delta \mathrm{H}_{\mathrm{f}}(\mathrm{kcal} / \mathrm{mol})=121.92, \Delta \mathrm{S}(\mathrm{cal} / \mathrm{molK})=68.67, \Delta \mathrm{G}_{\mathrm{f}}(\mathrm{kcal} / \mathrm{mol})=101.46$.

$\mathbf{H}_{2} \mathbf{O}\left(\right.$ PM5) : $\Delta \mathrm{H}_{\mathrm{f}}(\mathrm{kcal} / \mathrm{mol})=-59.47, \Delta \mathrm{S}(\mathrm{cal} / \mathrm{molK})=44.99, \Delta \mathrm{G}_{\mathrm{f}}(\mathrm{kcal} / \mathrm{mol})=-72.88$.

$\mathbf{H}_{3} \mathbf{O}^{+}(\mathbf{P M 5}): \Delta \mathrm{H}_{\mathrm{f}}(\mathrm{kcal} / \mathrm{mol})=46.51, \Delta \mathrm{S}(\mathrm{cal} / \mathrm{molK})=45.88, \Delta \mathrm{G}_{\mathrm{f}}(\mathrm{kcal} / \mathrm{mol})=32.84$.

Table 2. Aqueous phase ab initio calculated thermal and solvation free energies, investigated compounds at $298 \mathrm{~K}$

\begin{tabular}{|c|c|c|c|c|c|c|}
\hline & $\begin{array}{l}\text { Thermal } \\
\text { free } \\
\text { energy } \\
\text { Gg } \\
\mathrm{kcal} / \mathrm{mol}\end{array}$ & $\begin{array}{c}\text { Solvation } \\
\text { free } \\
\text { energy } \\
\mathrm{G}_{\mathrm{s}(\mathrm{B})} \\
\mathrm{kcal} / \mathrm{mol}\end{array}$ & $\begin{array}{c}\text { Total } \\
\text { solvation } \\
\text { free energy } \\
{ }^{\mathrm{a}} \Delta \mathrm{G}_{\mathrm{s}(\mathrm{B})} \\
\mathrm{kcal} / \mathrm{mol}\end{array}$ & $\begin{array}{c}\text { Thermal } \\
\text { free energy } \\
\mathrm{Gg}_{\left(\mathrm{BH}^{+}\right)} \\
\mathrm{kcal} / \mathrm{mol}\end{array}$ & $\begin{array}{c}\text { Solvation } \\
\text { free energy } \\
\mathrm{G}_{\mathrm{s}\left(\mathrm{BH}^{+}\right)} \\
\mathrm{kcal} / \mathrm{mol}\end{array}$ & $\begin{array}{c}\text { Total solvation free } \\
\text { energy }{ }^{b} \Delta \mathrm{G}_{\mathrm{s}\left(\mathrm{BH}^{+}\right)} \\
\mathrm{kcal} / \mathrm{mol}\end{array}$ \\
\hline $\begin{array}{c}\text { Comp } \\
\text { ound }\end{array}$ & & & & $\mathrm{F} / 6-31 \mathrm{G}(\mathrm{d})$ & & \\
\hline $1 \mathrm{~s}$ & $\begin{array}{c}- \\
308884.02\end{array}$ & $\begin{array}{c}- \\
308893.22\end{array}$ & -9.20 & & & \\
\hline $\begin{array}{l}\text { 1sNp } \\
1 \text { sN'p }\end{array}$ & & & & $\begin{array}{l}-309135.34 \\
-309135.34\end{array}$ & $\begin{array}{l}-309181.87 \\
-309181.87\end{array}$ & $\begin{array}{l}-46.53 \\
-46.53\end{array}$ \\
\hline
\end{tabular}


Table 2. Continued

\begin{tabular}{|c|c|c|c|c|c|c|}
\hline & $\begin{array}{l}\text { Thermal } \\
\text { Free } \\
\text { Energy } \\
\text { Gg } \\
\mathrm{kcal} / \mathrm{mol} \\
\end{array}$ & $\begin{array}{l}\text { Solvation } \\
\text { Free } \\
\text { Energy } \\
\mathrm{G}_{\mathrm{s}(\mathrm{B})} \\
\mathrm{kcal} / \mathrm{mol} \\
\end{array}$ & $\begin{array}{c}\text { Total } \\
\text { Solvation } \\
\text { Free Energy } \\
{ }^{\mathrm{a}} \Delta \mathrm{G}_{\mathrm{s}(\mathrm{B})} \\
\mathrm{kcal} / \mathrm{mol} \\
\end{array}$ & $\begin{array}{c}\text { Thermal } \\
\text { Free Energy } \\
\mathrm{Gg}_{\left(\mathrm{BH}^{+}\right)} \\
\mathrm{kcal} / \mathrm{mol}\end{array}$ & $\begin{array}{c}\text { Solvation } \\
\text { Free Energy } \\
\mathrm{G}_{\mathrm{s}\left(\mathrm{BH}^{+}\right)} \\
\mathrm{kcal} / \mathrm{mol}\end{array}$ & $\begin{array}{c}\text { Total } \\
\text { Solvation } \\
\text { Free Energy } \\
{ }^{\mathrm{b}} \Delta \mathrm{G}_{\mathrm{s}\left(\mathrm{BH}^{+}\right)} \\
\mathrm{kcal} / \mathrm{mol} \\
\end{array}$ \\
\hline $\begin{array}{l}\text { Comp } \\
\text { ound }\end{array}$ & \multicolumn{6}{|c|}{$\mathrm{HF} / 6-31 \mathrm{G}(\mathrm{d})$} \\
\hline $1 t$ & $\begin{array}{c}- \\
308890.59\end{array}$ & $\begin{array}{c}- \\
308895.81\end{array}$ & -5.21 & & & \\
\hline $\begin{array}{l}\text { 1tNp } \\
1 \text { tN'p }\end{array}$ & & & & $\begin{array}{l}-309128.56 \\
-309128.56\end{array}$ & $\begin{array}{l}-309179.72 \\
-309184.42\end{array}$ & $\begin{array}{l}-51.16 \\
-55.85\end{array}$ \\
\hline $2 s$ & $\begin{array}{c}- \\
308886.42\end{array}$ & $\begin{array}{c}- \\
308893.93\end{array}$ & -7.51 & & & \\
\hline $\begin{array}{l}2 \mathrm{sNp} \\
2 \mathrm{sN} \text { 'p }\end{array}$ & & & & $\begin{array}{l}-309123.87 \\
-309127.66\end{array}$ & $\begin{array}{l}-309178.15 \\
-309179.22\end{array}$ & $\begin{array}{l}-54.27 \\
-51.55\end{array}$ \\
\hline $2 t$ & $\begin{array}{c}- \\
308887.06\end{array}$ & $\begin{array}{c}- \\
308893.84\end{array}$ & -6.78 & & & \\
\hline $\begin{array}{c}2 \mathrm{tNp} \\
2 \mathrm{tN} \mathrm{N}^{\prime} \mathrm{p}\end{array}$ & & & & $\begin{array}{l}-309123.54 \\
-309126.25\end{array}$ & $\begin{array}{l}-309177.55 \\
-309178.81\end{array}$ & $\begin{array}{l}-54.01 \\
-52.55\end{array}$ \\
\hline 3 & $\begin{array}{c}- \\
308886.77\end{array}$ & $\begin{array}{c}- \\
308898.03\end{array}$ & -11.26 & & & \\
\hline $\begin{array}{l}3 \mathbf{N p} \\
3 N^{\prime} p\end{array}$ & & & & $\begin{array}{l}-309121.38 \\
-309128.99\end{array}$ & $\begin{array}{l}-309177.46 \\
-309179.92\end{array}$ & $\begin{array}{l}-56.07 \\
-50.93\end{array}$ \\
\hline $4 s$ & $\begin{array}{c}- \\
308884.51\end{array}$ & $\begin{array}{c}- \\
308900.95\end{array}$ & -16.44 & & & \\
\hline $\begin{array}{l}4 \mathrm{sNp} \\
4 \mathrm{sN} \text { 'p }\end{array}$ & & & & $\begin{array}{l}-309119.88 \\
-309119.88\end{array}$ & $\begin{array}{l}-309176.90 \\
-309181.47\end{array}$ & $\begin{array}{l}-57.02 \\
-61.59\end{array}$ \\
\hline $4 t$ & $\begin{array}{c}- \\
308884.65\end{array}$ & $\begin{array}{c}- \\
308901.09\end{array}$ & -16.44 & & & \\
\hline $\begin{array}{r}4 t \mathbf{t p p} \\
4 \mathrm{tN} ' p\end{array}$ & & & & $\begin{array}{l}-309119.67 \\
-309119.67\end{array}$ & $\begin{array}{l}-309181.09 \\
-309181.09\end{array}$ & $\begin{array}{l}-61.42 \\
-61.42\end{array}$ \\
\hline $\begin{array}{l}\text { Comp } \\
\text { ound }\end{array}$ & & & & $6-31 \mathrm{G}(\mathrm{d})$ & & \\
\hline 5 & $\begin{array}{c}- \\
308885.03\end{array}$ & $\begin{array}{c}- \\
308893.12\end{array}$ & -8.09 & & & \\
\hline $\begin{array}{c}5 \mathrm{~Np} \\
5 \mathrm{~N} \text { 'p }\end{array}$ & & & & $\begin{array}{l}-309118.31 \\
-309123.12\end{array}$ & $\begin{array}{l}-309176.60 \\
-309178.01\end{array}$ & $\begin{array}{l}-58.28 \\
-54.89\end{array}$ \\
\hline
\end{tabular}


Table 2. Continued

\begin{tabular}{|c|c|c|c|c|c|c|}
\hline & $\begin{array}{c}\text { Thermal } \\
\text { Free } \\
\text { Energy } \\
\text { Gg } \\
\mathrm{kcal} / \mathrm{mol}\end{array}$ & $\begin{array}{c}\text { Solvation } \\
\text { Free } \\
\text { Energy } \\
\mathrm{G}_{\mathrm{s}(\mathrm{B})} \\
\mathrm{kcal} / \mathrm{mol}\end{array}$ & $\begin{array}{c}\text { Total } \\
\text { Solvation } \\
\text { Free Energy } \\
{ }^{\mathrm{a}} \Delta \mathrm{G}_{\mathrm{s}(\mathrm{B})} \\
\mathrm{kcal} / \mathrm{mol}\end{array}$ & $\begin{array}{c}\text { Thermal } \\
\text { Free Energy } \\
\mathrm{Gg}_{\left(\mathrm{BH}^{+}\right)} \\
\mathrm{kcal} / \mathrm{mol}\end{array}$ & $\begin{array}{c}\text { Solvation } \\
\text { Free Energy } \\
\mathrm{G}_{\mathrm{s}\left(\mathrm{BH}^{+}\right)} \\
\mathrm{kcal} / \mathrm{mol}\end{array}$ & $\begin{array}{c}\text { Total } \\
\text { Solvation } \\
\text { Free Energy } \\
{ }^{\mathrm{b}} \Delta \mathrm{G}_{\mathrm{s}\left(\mathrm{BH}^{+}\right)} \\
\mathrm{kcal} / \mathrm{mol}\end{array}$ \\
\hline $\begin{array}{l}\text { Comp } \\
\text { ound }\end{array}$ & \multicolumn{6}{|c|}{$\mathrm{HF} / 6-31 \mathrm{G}(\mathrm{d})$} \\
\hline 6 & $\begin{array}{c}- \\
308885.17\end{array}$ & $\begin{array}{c}- \\
308893.62\end{array}$ & -8.45 & & & \\
\hline $\begin{array}{l}\text { 6Np } \\
\text { 6N'p }\end{array}$ & & & & $\begin{array}{l}-309120.48 \\
-309120.48\end{array}$ & $\begin{array}{l}-309177.41 \\
-309177.41\end{array}$ & $\begin{array}{l}-56.92 \\
-56.92\end{array}$ \\
\hline
\end{tabular}

Table 3. Aqueous phase ab initio calculated thermal and solvation free energies, investigated compounds at $298 \mathrm{~K}$

\begin{tabular}{|c|c|c|c|c|c|c|}
\hline & $\begin{array}{l}\text { Thermal } \\
\text { Free } \\
\text { Energy } \\
\text { Gg } \\
\text { kcal/mol }\end{array}$ & $\begin{array}{l}\text { Solvation } \\
\text { Free } \\
\text { Energy } \\
{ }^{\mathrm{a}} \Delta \mathrm{G}_{\mathrm{s}(\mathrm{B})} \\
\mathrm{kcal} / \mathrm{mol}\end{array}$ & $\begin{array}{c}\text { Total } \\
\text { Solvation } \\
\text { Free } \\
\text { Energy } \\
{ }^{\mathrm{a}} \Delta \mathrm{G}_{\mathrm{s}(\mathrm{B})} \\
\mathrm{kcal} / \mathrm{mol} \\
\end{array}$ & $\begin{array}{l}\text { Thermal } \\
\text { Free } \\
\text { Energy } \\
\mathrm{Gg}_{\left(\mathrm{BH}^{+}\right)} \\
\mathrm{kcal} / \mathrm{mol}\end{array}$ & $\begin{array}{c}\text { Solvation } \\
\text { Free } \\
\text { Energy } \\
\mathrm{G}_{\mathrm{s}\left(\mathrm{BH}^{+}\right)} \\
\mathrm{kcal} / \mathrm{mol}\end{array}$ & $\begin{array}{c}\text { Total } \\
\text { Solvation } \\
\text { Free } \\
\text { Energy } \\
{ }^{\mathrm{b}} \Delta \mathrm{G}_{\mathrm{s}\left(\mathrm{BH}^{+}\right)} \\
\mathrm{kcal} / \mathrm{mol} \\
\end{array}$ \\
\hline $\begin{array}{l}\text { Comp } \\
\text { ound }\end{array}$ & \multicolumn{6}{|c|}{ B3LYP/6-31G(d) } \\
\hline $1 \mathrm{~s}$ & -310853.47 & -310868.95 & -15.48 & & & \\
\hline $1 \mathrm{sNp}$ & & & & -311105.14 & -311154.23 & -49.08 \\
\hline 1sN'p & & & & -311105.14 & -311154.23 & -49.08 \\
\hline $1 \mathrm{t}$ & -310860.44 & -310866.68 & -6.24 & & & \\
\hline $1 t N p$ & & & & -311097.79 & -311150.30 & -52.51 \\
\hline 1tN'p & & & & -311097.79 & -311149.05 & -51.26 \\
\hline $2 \mathrm{~s}$ & -310856.23 & -310864.90 & -8.67 & & & \\
\hline $2 \mathrm{sNp}$ & & & & -311092.66 & -311146.05 & -53.39 \\
\hline $2 \mathrm{sN}$ 'p & & & & -311097.21 & -311150.72 & -53.50 \\
\hline $2 t$ & -310856.93 & -310865.66 & -8.73 & & & \\
\hline $2 \mathrm{tNp}$ & & & & -311092.27 & -311145.57 & -53.30 \\
\hline 2tN'p & & & & -311095.65 & -311148.90 & -53.24 \\
\hline
\end{tabular}


Table 3. Continued

\begin{tabular}{|c|c|c|c|c|c|c|}
\hline & $\begin{array}{c}\text { Thermal } \\
\text { Free } \\
\text { Energy } \\
\text { Gg } \\
\text { kcal/mol }\end{array}$ & $\begin{array}{l}\text { Solvation } \\
\text { Free } \\
\text { Energy } \\
{ }^{\mathrm{a}} \Delta \mathrm{G}_{\mathrm{s}(\mathrm{B})} \\
\mathrm{kcal} / \mathrm{mol}\end{array}$ & $\begin{array}{c}\text { Total } \\
\text { Solvation } \\
\text { Free } \\
\text { Energy } \\
{ }^{\mathrm{a}} \Delta \mathrm{G}_{\mathrm{s}(\mathrm{B})} \\
\mathrm{kcal} / \mathrm{mol} \\
\end{array}$ & $\begin{array}{c}\text { Thermal } \\
\text { Free } \\
\text { Energy } \\
\mathrm{Gg}_{\left(\mathrm{BH}^{+}\right)} \\
\mathrm{kcal} / \mathrm{mol}\end{array}$ & $\begin{array}{c}\text { Solvation } \\
\text { Free } \\
\text { Energy } \\
\mathrm{G}_{\mathrm{s}\left(\mathrm{BH}^{+}\right)} \\
\mathrm{kcal} / \mathrm{mol}\end{array}$ & $\begin{array}{c}\text { Total } \\
\text { Solvation } \\
\text { Free } \\
\text { Energy } \\
{ }^{\mathrm{b}} \Delta \mathrm{G}_{\mathrm{s}\left(\mathrm{BH}^{+}\right)} \\
\mathrm{kcal} / \mathrm{mol} \\
\end{array}$ \\
\hline $\begin{array}{l}\text { Comp } \\
\text { ound }\end{array}$ & \multicolumn{6}{|c|}{ B3LYP/6-31G(d) } \\
\hline 3 & -310856.78 & -310868.51 & -11.72 & & & \\
\hline $3 N p$ & & & & -311090.43 & -311149.70 & -59.27 \\
\hline $3 N^{\prime} p$ & & & & -311098.66 & -311149.39 & -50.72 \\
\hline $4 s$ & -310854.58 & -310869.79 & -15.21 & & & \\
\hline $4 \mathrm{sNp}$ & & & & -311089.32 & -311144.54 & -55.22 \\
\hline $4 s N^{\prime} p$ & & & & -311089.32 & -311144.54 & -55.22 \\
\hline $4 t$ & -310854.72 & -310870.24 & -15.52 & & & \\
\hline $4 \mathrm{tNp}$ & & & & -311089.09 & -311148.73 & -59.64 \\
\hline $4 \mathrm{tN}$ 'p & & & & -311089.09 & -311148.72 & -59.64 \\
\hline 5 & -310854.87 & -310859.15 & -4.28 & & & \\
\hline $5 \mathrm{~Np}$ & & & & -311087.55 & -311141.45 & -53.89 \\
\hline $5 N^{\prime} p$ & & & & -311092.12 & -311143.24 & -51.11 \\
\hline 6 & -310854.93 & -310871.68 & -16.75 & & & \\
\hline $6 \mathrm{~Np}$ & & & & -311089.76 & -311148.65 & -58.88 \\
\hline 6N'p & & & & -311089.76 & -311148.65 & -58.88 \\
\hline
\end{tabular}

Table 4. Aqueous phase PM5 and ab initio calculated $\mathrm{pK}_{\mathrm{a}}$ values for the investigated molecules

\begin{tabular}{|c|c|c|c|c|c|}
\hline $\begin{array}{c}\text { Protonation } \\
\text { pattern }\end{array}$ & $\begin{array}{c}\delta \Delta \mathrm{G}_{\mathrm{f}\left(\mathrm{BH}^{+}\right)} \\
(\mathrm{kcal} / \mathrm{mol})^{\mathrm{a}}\end{array}$ & $\mathrm{pKa}^{\mathrm{b}}$ & $\mathrm{pKa}^{\mathrm{c}}$ & $\mathrm{pKa}^{\mathrm{d}}$ & $\mathrm{pKa}(\exp .)^{\mathrm{e}}$ \\
\hline $1 \mathrm{~s} \leftrightarrow 1 \mathrm{sN} \mathbf{N}^{\prime} \mathrm{p}$ & 4.71 & 3.45 & 12.80 & 10.32 & 4.44 \\
\hline $1 \mathrm{~s} \leftrightarrow 1 \mathrm{sNp}$ & 4.74 & 3.48 & 12.80 & 10.32 & \\
\hline $2 s \leftrightarrow 2 s N^{\prime} p$ & 4.57 & 3.35 & 10.33 & 10.71 & 4.42 \\
\hline $2 \mathrm{~s} \leftrightarrow 2 \mathrm{sNp}$ & 2.52 & 1.85 & 9.54 & 7.30 & \\
\hline $3 \leftrightarrow 3 N^{\prime} p$ & 5.41 & 3.97 & 7.84 & 7.10 & 4.77 \\
\hline $3 \leftrightarrow 3 \mathrm{~Np}$ & 2.15 & 1.58 & 6.03 & 7.33 & \\
\hline $4 s \leftrightarrow 4 s N^{\prime} p$ & 5.08 & 3.72 & 6.83 & 2.60 & 4.60 \\
\hline $4 \mathrm{~s} \leftrightarrow 4 \mathrm{sNp}$ & 4.97 & 3.65 & 3.48 & 2.60 & \\
\hline $5 \leftrightarrow 5 N^{\prime} p$ & 5.85 & 4.29 & 10.04 & 9.45 & 4.85 \\
\hline
\end{tabular}


Table 4. Continued

\begin{tabular}{cccccc}
\hline $\begin{array}{c}\text { Protonation } \\
\text { pattern }\end{array}$ & $\begin{array}{c}\delta \Delta \mathrm{G}_{\mathrm{f}\left(\mathrm{BH}^{+}\right)} \\
(\mathrm{kcal} / \mathrm{mol})^{\mathrm{a}}\end{array}$ & $\mathrm{pKa}^{\mathrm{b}}$ & $\mathrm{pKa}^{\mathrm{c}}$ & $\mathrm{pKa}^{\mathrm{d}}$ & $\mathrm{pKa}(\exp .)^{\mathrm{e}}$ \\
\hline $\mathbf{5} \mathbf{5} \mathbf{N p}$ & 4.20 & 3.08 & 9.00 & 8.13 & \\
$\mathbf{6} \boldsymbol{6} \mathbf{6} \mathbf{N}^{\prime} \mathbf{p}$ & 5.78 & 4.24 & 9.23 & 4.22 & 4.82 \\
$\mathbf{6} \leftrightarrow \mathbf{6 N p}$ & 5.77 & 4.23 & 9.23 & 4.22 & \\
\hline
\end{tabular}

${ }^{\mathrm{a}} \delta \Delta \mathrm{G}_{f\left(\mathrm{BH}^{+}\right)}=\left[\Delta \mathrm{G}_{f(\mathrm{~B})}+\Delta \mathrm{G}_{f\left(\mathrm{H}_{3} \mathrm{O}^{+}\right)}\right]-\left[\Delta \mathrm{G}_{f\left(\mathrm{BH}^{+}\right)}+\Delta \mathrm{G}_{f(\mathrm{H} 2 \mathrm{O})}\right] .{ }^{\mathrm{b}} \mathrm{pK}_{\mathrm{a}}=\delta \Delta \mathrm{G}_{f\left(\mathrm{BH}^{+}\right)} /(2.303 \mathrm{RT}) .{ }^{\mathrm{c}} \mathrm{pK} \mathrm{a}_{\mathrm{a}}$ calculated HF /6-31G(d) cisoid and transoid form. ${ }^{\mathrm{d}} \mathrm{pK}_{\mathrm{a}}$ calculated B3LYP /6-31G(d) cisoid and transoid form. ${ }^{\mathrm{e}} \mathrm{pK}_{\mathrm{a} \text { (expt.) }}$ were taken from ref. 45 , PM5 Calculated $\mathrm{pK}_{\mathrm{a}}$ value for pyridine is 5.10

Table 5. Aqueous phase PM5 and ab initio calculated $\mathrm{pK}_{\mathrm{a}}$ values for the investigated molecules

\begin{tabular}{|c|c|c|c|c|c|}
\hline $\begin{array}{c}\text { Protonation } \\
\text { pattern }\end{array}$ & $\begin{array}{c}\delta \Delta \mathrm{G}_{\mathrm{f}\left(\mathrm{BH}^{+}\right)} \\
(\mathrm{kcal} / \mathrm{mol})^{\mathrm{a}}\end{array}$ & $\mathrm{pKa}^{\mathrm{b}}$ & $\mathrm{pKa}^{\mathrm{c}}$ & $\mathrm{pKa}^{\mathrm{d}}$ & $\mathrm{pKa}(\exp .)^{\mathrm{e}}$ \\
\hline $1 t \leftrightarrow 1 t N^{\prime} p$ & 2.90 & 2.13 & 12.77 & 8.19 & 4.44 \\
\hline $1 \mathrm{t} \leftrightarrow 1 \mathrm{tNp}$ & 2.83 & 2.07 & 9.33 & 9.11 & \\
\hline $2 \mathrm{t} \leftrightarrow 2 \mathrm{t} \mathrm{N}^{\prime} p$ & 4.38 & 3.21 & 10.09 & 8.82 & 4.42 \\
\hline $2 \mathrm{t} \leftrightarrow 2 \mathrm{tNp}$ & 2.88 & 2.11 & 9.17 & 6.39 & \\
\hline $\begin{array}{c}3 \leftrightarrow 3 N^{\prime} p \\
3 \leftrightarrow 3 N p\end{array}$ & \multicolumn{5}{|c|}{ No cisoid or transoid form } \\
\hline $4 \mathrm{t} \leftrightarrow 4 \mathrm{t} \mathrm{N}^{\prime} p$ & 5.10 & 3.74 & 6.45 & 5.35 & 4.60 \\
\hline $4 \mathrm{t} \leftrightarrow 4 \mathrm{tNp}$ & 5.09 & 3.73 & 6.45 & 5.35 & \\
\hline $\begin{array}{l}5 \leftrightarrow 5 N^{\prime} p \\
5 \leftrightarrow 5 N p\end{array}$ & \multicolumn{5}{|c|}{ No cisoid or transoid form } \\
\hline $\begin{array}{c}6 \leftrightarrow 6 N^{\prime} p \\
6 \leftrightarrow 6 N^{\prime} p\end{array}$ & \multicolumn{5}{|c|}{ No cisoid or transoid form } \\
\hline
\end{tabular}

${ }^{\mathrm{a}} \delta \Delta \mathrm{G}_{f\left(\mathrm{BH}^{+}\right)}=\left[\Delta \mathrm{G}_{f(\mathrm{~B})}+\Delta \mathrm{G}_{f\left(\mathrm{H}_{3} \mathrm{O}^{+}\right)}\right]-\left[\Delta \mathrm{G}_{f\left(\mathrm{BH}^{+}\right)}+\Delta \mathrm{G}_{f(\mathrm{H} 2 \mathrm{O})}\right] .{ }^{\mathrm{b}} \mathrm{pK}_{\mathrm{a}}=\delta \Delta \mathrm{G}_{f\left(\mathrm{BH}^{+}\right)} /(2.303 \mathrm{RT}) .{ }^{\mathrm{c}} \mathrm{pK} \mathrm{a}_{\mathrm{a}}$ calculated HF /6-31G(d) cisoid and transoid form. ${ }^{\mathrm{d}} \mathrm{pK}_{\mathrm{a}}$ calculated B3LYP /6-31G(d) cisoid and transoid form. ${ }^{\mathrm{e}} \mathrm{pK}_{\mathrm{a} \text { (expt.) }}$ were taken from ref. 45 , PM5 Calculated $\mathrm{pK}_{\mathrm{a}}$ value for pyridine is 5.10

Possible protonation patterns for the studied molecules were depicted in Scheme 2. The aqueous phase calculated acidity constants, $\mathrm{pK}_{\mathrm{a}}$ values, were given in Table 4and 5. From these data the $\mathrm{pK}_{\mathrm{a}}$ value of compound 5 was found to be the highest (i.e. 4.29 by PM5) where as the lowest $\mathrm{pK}_{\mathrm{a}}$ value of 3.35 was found for compound 2 . The following sequence of decreasing basicity was obtained;

$\begin{array}{llllllll}\text { Molecule } & : & 5 & 6 & 3 & 4 & 1 & 2\end{array}$

Experimental $\mathrm{pK}_{\mathrm{a}}$ : $\quad$ 3,4'-bpy. 4,4'-bpy. 2,4'-bpy. 3,3'-bpy. 2,2'-bpy. 2,3'-bpy 


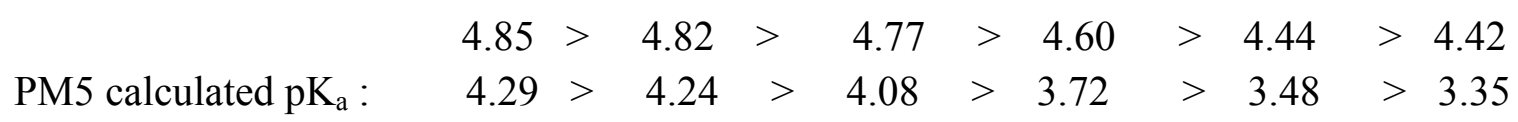

basicity decrease

acidity increase

A more reliable and closer $\mathrm{pK}_{\mathrm{a}}$ values to experimental values however were found by $a b$ initio calculations (Table 4 and 5) for compound $\mathbf{3}$.

A perfect correlation was observed between theoretically obtained (i.e. PM5 calculated) and experimental acidity constants [46] with a slope and regression of about unity (i.e. $\mathrm{R}^{2}=0.99$ ). This high correlation indicates that the first protonation of compounds $\mathbf{1}$ and $\mathbf{3}$ occur over the cisoid form (i.e. they are off the line ). Whereas the first protonation of compound $\mathbf{4}$ occur over both cisoid and transoid form which are exist in equal amounts (Table 6). On the other hand protonation of compounds $\mathbf{2 , 5}$ and $\mathbf{6}$ occur over transoid form(i.e. they are on the same line)

A graph of experimental $\mathrm{pK}_{\mathrm{a}}$ values against the calculated $\mathrm{pK}_{\mathrm{a}}$ values using Equation 5 were depicted in Figure 1.

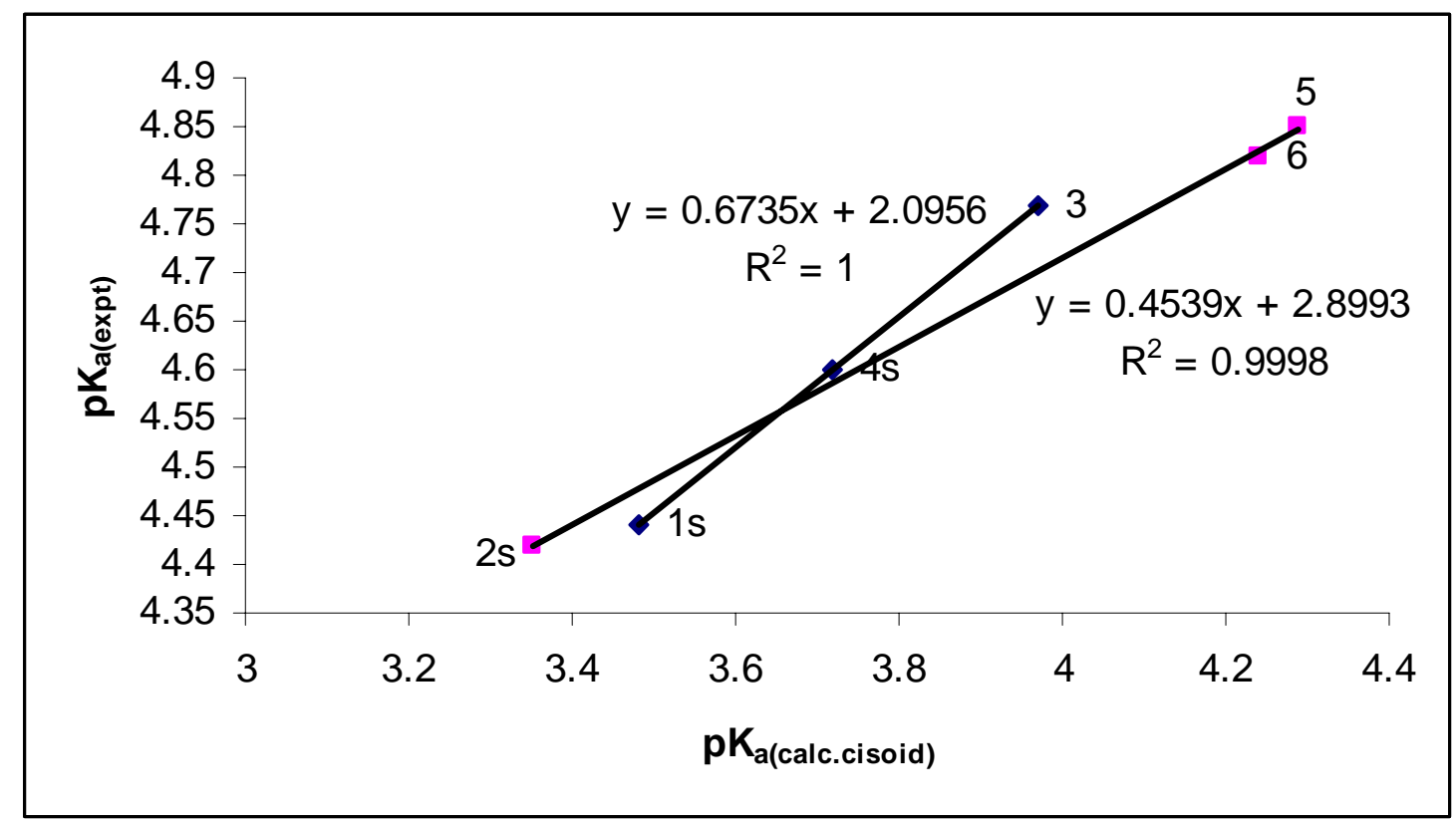

Figure 1. The plot of the aqueous phase PM5, calculated acidity constants, $\mathrm{pK}_{\mathrm{a}(\text { calc.cisoid), }}$, and experimental acidity constants, $\mathrm{pK}_{\mathrm{a}(\text { expt.), }}$, for bipyridine derivatives. 


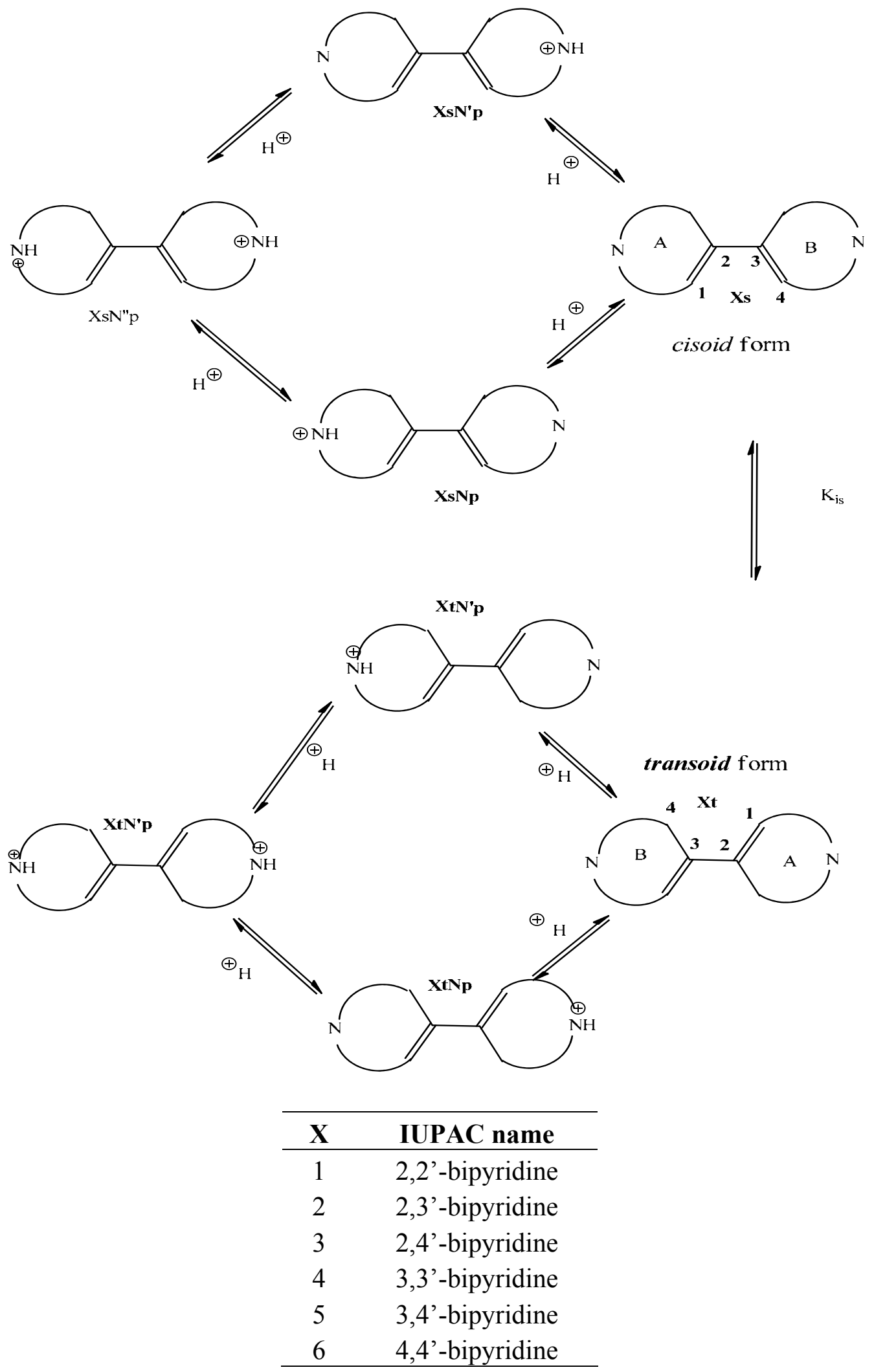

Scheme 2. Possible stereoisomerism and protonation pattern for studied molecules. 


\section{Substituent constants}

One of the commonly used method to search for the effect of substituent on an equilibrium processes has been the usage of the Hammett Equation (Equation 7). ${ }^{45}$

$$
\left.\mathrm{pK}_{\mathrm{a}} \text { (unsubstituted molecule }\right)-\mathrm{pK}_{\mathrm{a}}(\text { substituted molecule })=\rho \sigma
$$

The $\sigma$ (i.e. substituent constant) values for 2-, 3- and 4- pyridinyl group can be calculated by using Equation 7. The $\rho$ (i.e. equilibrium constant) value for the pyridine protonation was given as $5.77{ }^{46}$ Hence the ionization equilibrium in water for protonated pyridinium molecules can be written as in Scheme 3.The experimental $\mathrm{pK}_{\mathrm{a}}$ value for unsubstituted pyridine was taken as $5.17 .^{46}$

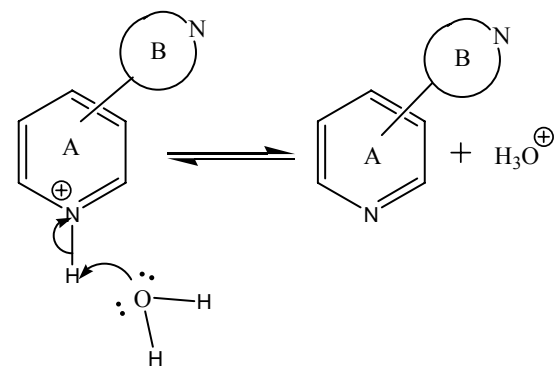

B = 2-(2'- pyridyl, 3'-pyridyl, 4'-pyridyl); 3-(3'- pyridyl, 4'-pyridyl) ; 4-(4'-pyridyl)

Scheme 3. Deprotonation pattern for pyridinium ionization in water.

The substituent constants using the experimental and theoretical $\mathrm{pK}_{\mathrm{a}}$ values were calculated and obtained results were depicted in Table 7. When we take $\sigma$ values of which calculated using the experimental $\mathrm{pK}_{\mathrm{a}}$ values into account we observed that all $\sigma$ values have positive sign indicating the electron -withdrawing effect of the substituted pyridyl ring (B).

We also can predict that this electron-withdrawing effect is not big and it is close to the $\sigma_{\mathrm{p}}$ value of 0.06 for fluorine atom. ${ }^{47}$ It seems that the obtained calculation results are quite satisfactory and PM5 calculated $\sigma$ values for all studied molecules were close enough to experimental values to be considered successful.

\section{Relative Stabilities}

Equation 8 was used to calculate the relative stabilities (RS) using the heat of formation value of the studied molecules.

$$
\mathrm{RS}=\Delta \mathrm{H}_{\mathrm{f}}(\text { transoid form })-\Delta \mathrm{H}_{\mathrm{f}}(\text { cisoid form })
$$

The aqueous phase PM5 calculated relative stabilities were given in Table 6.The RS values 
indicate that the transoid form is predominant over the cisoid form for molecule $\mathbf{1}$ and $\mathbf{2}$. RS values for 3, $\mathbf{5}$ and $\mathbf{6}$ indicate equal amount of cisoid and transoid forms, as expected, because of the symmetry. On the other hand the PM5 calculated RS values are indicative of equal amount of cisoid and transoid forms for compound $\mathbf{4}$. Whereas the ab initio calculated RS values suggest the predominance of cisoid forms.

Table 6. The aqueous phase PM5, HF and B3LYP calculated isomeric equilibrium constants $\left(\mathrm{K}_{\mathrm{is}}\right)$ stability constants $(\mathrm{RS})$ and mole fraction $(\mathrm{N})$ values of studied molecules

\begin{tabular}{|c|c|c|c|c|c|c|c|c|}
\hline $\begin{array}{l}\text { Process } \\
\text { Isomerism }\end{array}$ & $\begin{array}{c}\delta \Delta \mathrm{G} \\
(\mathrm{kcal} / \mathrm{mol} \\
)^{\mathrm{a}}\end{array}$ & $\mathrm{K}_{\mathrm{is}}^{\mathrm{b}}$ & $\mathrm{N}_{\mathrm{s}}^{\mathrm{c}}$ & $\mathrm{N}_{\mathrm{t}}^{\mathrm{c}}$ & $\mathrm{K}_{\mathrm{is}}{ }^{\mathrm{d}}$ & $\mathrm{N}_{\mathrm{t}}^{\mathrm{e}}$ & $\mathrm{RS}^{\mathrm{f}}$ & $\begin{array}{l}\text { Suggested } \\
\text { Confirmation }\end{array}$ \\
\hline \multicolumn{9}{|l|}{ PM5 } \\
\hline $1 s-1 t$ & -0.05 & 1.09 & 0.48 & 0.52 & 22.38 & $\begin{array}{l}0.04 \\
0.96\end{array}$ & 0.10 & Transoid $^{\mathrm{b}, \mathrm{c}, \mathrm{d}, \mathrm{e}, \mathrm{f}}$ \\
\hline $2 s-2 t$ & 0.16 & 0.76 & 0.57 & 0.43 & 1.38 & $\begin{array}{l}0.42 \\
0.58\end{array}$ & 0.18 & Transoid $_{\mathrm{b}, \mathrm{c}}^{\mathrm{de,}, \mathrm{f}}$ Cisoid \\
\hline $4 s-4 t$ & 0.00 & 1.00 & 0.50 & 0.50 & 0.95 & $\begin{array}{l}0.51 \\
0.49\end{array}$ & 0.01 & Equal amount $t^{\mathrm{b}, \mathrm{c}, \mathrm{d}, \mathrm{e}, \mathrm{f}}$ \\
\hline \multicolumn{9}{|l|}{ HF/6-31G(d) } \\
\hline $1 s-1 t$ & -2.58 & 78.10 & 0.01 & 0.99 & 2951 & $\begin{array}{l}0.01 \\
0.99\end{array}$ & -6.57 & $\begin{array}{c}\text { Transoid }^{\mathrm{b}, \mathrm{c}, \mathrm{d}, \mathrm{e}} \\
\text { Cisoid }^{\mathrm{f}}\end{array}$ \\
\hline $2 s-2 t$ & 0.09 & 0.85 & 0.54 & 0.46 & 2.34 & $\begin{array}{l}0.30 \\
0.70\end{array}$ & -0.63 & $\underset{\mathrm{b}, \mathrm{c}, \mathrm{f}}{\text { Transoid }^{\mathrm{d}, \mathrm{e}}}$ Cisoid \\
\hline $4 s-4 t$ & -0.14 & 1.26 & 0.45 & 0.55 & 0.001 & $\begin{array}{l}0.99 \\
0.01\end{array}$ & -0.14 & $\underset{\mathrm{d}, \mathrm{e}, \mathrm{f}}{\text { Transoid }^{\mathrm{b}, \mathrm{c},} \text { Cisoid }}$ \\
\hline \multicolumn{9}{|l|}{$\begin{array}{l}\text { B3LYP/ } \\
\text { 6-31G(d) }\end{array}$} \\
\hline $1 s-1 t$ & 2.27 & 0.02 & 0.98 & 0.02 & 134.8 & $\begin{array}{l}0.01 \\
0.99\end{array}$ & -6.96 & $\begin{array}{c}\text { Transoid }^{\mathrm{b}, \mathrm{c}, \mathrm{d}, \mathrm{e}} \\
\text { Cisoid }^{\mathrm{f}}\end{array}$ \\
\hline $2 s-2 t$ & -0.75 & 3.59 & 0.22 & 0.78 & 8.12 & $\begin{array}{l}0.32 \\
0.68\end{array}$ & -0.70 & $\begin{array}{c}\text { Transoid }^{\mathrm{b}, \mathrm{c}, \mathrm{d}, \mathrm{e}} \\
\text { Cisoid }^{\mathrm{f}}\end{array}$ \\
\hline $4 s-4 t$ & -0.45 & 2.13 & 0.32 & 0.68 & 0.001 & $\begin{array}{l}0.99 \\
0.01\end{array}$ & -0.14 & $\underset{\mathrm{d}, \mathrm{e}, \mathrm{f}}{\text { Transoid }^{\mathrm{b}, \mathrm{c},}}$ Cisoid \\
\hline
\end{tabular}

${ }^{\mathrm{a}} \delta \Delta \mathrm{G}=\Delta \mathrm{G}($ product $)-\Delta \mathrm{G}($ reactant $)$.

${ }^{\mathrm{b}} \mathrm{K}_{\text {is }}=\mathrm{e}^{-(\delta \Delta \mathrm{G} / \mathrm{RT})}$.

${ }^{c} \mathrm{~N}_{\mathrm{s}}=1 /\left(1+\mathrm{K}_{\mathrm{is}}\right) \quad \mathrm{N}_{\mathrm{t}}=\mathrm{K}_{\mathrm{is}} /\left(1+\mathrm{K}_{\mathrm{is}}\right) \quad\left(\mathrm{K}_{\mathrm{is}}\right.$ values were calculated by using $\delta \Delta \mathrm{G}$ values).

${ }^{\mathrm{d}} \mathrm{K}_{\text {is }}=\mathrm{K}_{\mathrm{a}}$ (transoid) $/ \mathrm{K}_{\mathrm{a}}$ (cisoid).

${ }^{\mathrm{e}} \mathrm{N}_{\mathrm{s}}=1 /\left(1+\mathrm{K}_{\mathrm{is}}\right) \quad \mathrm{N}_{\mathrm{t}}=\mathrm{K}_{\text {is }} /\left(1+\mathrm{K}_{\text {is }}\right) \quad$ ( $\mathrm{K}_{\text {is }}$ values were calculated by utilizing the $\mathrm{pK}_{\mathrm{a}}$ values).

${ }^{\mathrm{f}} \mathrm{RS}=\Delta \mathrm{H}_{\mathrm{f}}$ (transoid) $-\Delta \mathrm{H}_{\mathrm{f}}($ cisoid $)$ The negative sign indicates the favorability of cisoid form. 
Table 7. Aqueous phase PM5, HF and B3LYP calculated substituent constants, $\sigma$ values, for investigated compounds

\begin{tabular}{ccccccc}
\hline Molecule & Substituent & Form & $\sigma_{\text {(calc.) }}{ }^{\mathrm{a}}$ & $\sigma_{\text {(calc.) }}{ }^{\mathrm{b}}$ & $\sigma_{\text {(calc.) }}{ }^{\mathrm{c}}$ & $\sigma_{\text {(expt.) }}$ \\
\hline \multirow{2}{*}{$\mathbf{1}$} & \multirow{2}{*}{ 2'-pyridine } & Cisoid & 0.29 & 0.68 & 1.49 & \\
& & Transoid & 0.53 & 0.08 & 0.09 & 0.13 \\
$\mathbf{2}$ & 3'-pyridine & Cisoid & 0.32 & 0.12 & 0.32 & \\
& & Transoid & 0.34 & 0.21 & 0.89 & 0.13 \\
$\mathbf{3}$ & 4'-pyridine & & 0.21 & -0.17 & -0.25 & 0.07 \\
$\mathbf{4}$ & 3'-pyridine & Cisoid & 0.25 & -0.34 & -1.07 & 0.10 \\
& & Transoid & 0.25 & -0.41 & -0.60 & \\
$\mathbf{5}$ & 4'-pyridine & & 0.15 & 0.03 & 0.10 & 0.06 \\
$\mathbf{6}$ & 4'-pyridine & & 0.16 & 0.06 & -0.79 & 0.06 \\
\hline
\end{tabular}

${ }^{a}$ Calculated using PM5 semiempiric $\mathrm{pK}_{\mathrm{a}}$ values. The plus sign indicates the electron-withdrawing effect.

${ }^{\mathrm{b}}$ Calculated using $\mathrm{HF} / 6-31 \mathrm{G}(\mathrm{d})$ calculated $\mathrm{pK}_{\mathrm{a}}$ values.

${ }^{c}$ Calculated using B3LYP/ 6-31 G (d) calculated $\mathrm{pK}_{\mathrm{a}}$ values.

${ }^{\mathrm{d}}$ Calculated using experimental $\mathrm{pK}_{\mathrm{a}}$ values using the Hammett equation $\mathrm{pK}_{\mathrm{a}}$ (unsubst. pyridine) $\mathrm{pK}_{\mathrm{a}}($ subst. pyridine $)=\rho \sigma ; \rho($ for pyridine protanation $)=5.77^{46}$

\section{Isomeric Equilibrium Constants}

The isomeric equilibrium constants, $\mathrm{K}_{\mathrm{is}}$ values, of the studied molecules were calculated by two methods as described below;

i- Using the equation $\mathrm{K}_{\mathrm{is}}=\mathrm{e}^{\left(\delta \Delta \mathrm{G}_{\mathrm{f}} / \mathrm{RT}\right)}$

Where $\mathrm{K}_{\mathrm{is}}$ is the isomeric equilibrium constant between the cisoid and transoid forms. $\delta \Delta \mathrm{G}_{\mathrm{f}}$ is the difference in Gibbs free energies of the isomers, $\delta \Delta \mathrm{G}_{\mathrm{f}}=\Delta \mathrm{G}_{\mathrm{f}}$ (transoid form) $-\Delta \mathrm{G}_{\mathrm{f}}$ (cisoid form). The $\mathrm{R}$ value is $1.987 \times 10^{-3} \mathrm{kcal} / \mathrm{mol}$ and $\mathrm{T}$ is $298 \mathrm{~K}$. The following equilibrium represents the isomeric equilibrium between isomers cisoid form and transoid form. The $\mathrm{K}_{\mathrm{is}}$ values obtained by this way suggest the predominance of transoid form over the cisoid forms for compounds 1 and 4. The predominance of cisoid form over the transoid form for compound 2. Whereas for compounds $\mathbf{3 , 5}$ and $\mathbf{6}$ they suggest the presence of the equal amount of cisoid and transoid forms.

$$
\text { cisoid form } \stackrel{\mathrm{K}_{\mathrm{is}}}{\rightleftharpoons} \text { transoid form }
$$


ii- $\quad$ Using the equations 6 and 7

$$
\begin{aligned}
& \mathrm{pK}_{\mathrm{a}}(\text { transoid })-\mathrm{pK}_{\mathrm{a}}(\text { cisoid })=\mathrm{pK}_{\mathrm{is}} \\
& \left.\mathrm{K}_{\mathrm{is}}=\mathrm{K}_{\mathrm{a}} \text { (transoid }\right) / \mathrm{K}_{\mathrm{a}}(\text { cisoid })
\end{aligned}
$$

The obtained $\mathrm{K}_{\text {is }}$ values by using Equations $9-10$ were depicted in Table 6 . The $\mathrm{K}_{\text {is }}$ values obtained using Equations 9-10, like the other $\mathrm{K}_{\text {is }}$ values mentioned above have suggested the predominance of the transoid form over the cisoid form for compound 1. Similarly for compound $\mathbf{2}$ the predominance of transoid form over the cisoid form for compound $\mathbf{2}$ was confirmed. For compound $\mathbf{4}$ supporting to above conclusion it indicates the predominance of the cisoid form over the transoid form. Whereas for compounds $\mathbf{3 , 5 , 6}$ it suggest the presence of the equal amount of cisoid and transoid forms. Ab initio calculated $\mathrm{K}_{\text {is }}$ values are however suggest the predominance of transoid forms with a values of 1.26 and 2.13 respectively (Table 6).

\section{Mole Fractions}

The mol fractions of the cisoid and transoid isomers were calculated by using the following equations and the obtained results were depicted in Table 6.

$\mathrm{N}($ cisoid form $)=1 /\left(1+\mathrm{K}_{\mathrm{is}}\right)$

$\mathrm{N}($ transoid form $)=\mathrm{K}_{\text {is. }} /\left(1+\mathrm{K}_{\mathrm{is}}\right)$

Mole fraction values indicate that the predominance of transoid form over the cisoid form for 1. For 2 however two different mole fraction calculation methods indicates two different conclusions; the first one, $\mathrm{N}^{\mathrm{c}}$ values, indicates the predominance of cisoid form(with the exception of the B3LYP method). Whereas the second one, $\mathrm{N}^{\mathrm{e}}$ values, indicates the predominance of transoid form. This discrepancy presumably source out from the difference between two methods of calculations. In the first one, $\mathrm{K}_{\mathrm{is}}{ }^{\mathrm{b}}$ values, the solvation effect is being neglected in the second one, $\mathrm{K}_{\text {is }}{ }^{\mathrm{d}}$ values, the solvation effect has been taken into account. This point had reflected also in the geometry of the neutral and protonated species in calculations of acidity constants, $\mathrm{pK}_{\mathrm{a}}$ values which were used in calculations of $\mathrm{N}^{\mathrm{e}}$ values. The change in the dihedral angle of N1-C2-C3-C4 ( i.e. changes from -53.74 to -0.06 for cisoid protonation and from -0.04 to -0.02 for transoid form) for cisoid protonation may explain why compound 2 prefers to exist in the transoid form (Table 8). For compound 4 the calculated mole fraction values leads to two differerent conclusions; $\mathrm{N}^{\mathrm{c}}$ values, indicates the predominance of transoid form. Whereas the other conclusions, $\mathrm{N}^{\mathrm{e}}$ values, indicates the predominances of cisoid form. For $\mathbf{3 , 5 , 6}$ however they suggest the existence of equal amount of two isomers (Table 6 ). 
Table 8. Aqueous phase PM5, HF and B3LYP calculated dihedral angles for studied neutral and protonated molecules

\begin{tabular}{|c|c|c|c|c|}
\hline & Molecule & Neutral & & First Protonation \\
\hline $\begin{array}{c}\text { Dihedral } \\
\text { Angels } \\
\end{array}$ & & \multicolumn{3}{|c|}{ cisoid } \\
\hline PM5 & & Xs & $\mathrm{XsNp}$ & XsN'p \\
\hline N1-C2-C3-N4 & 1 & -0.05 & 179.80 & 179.65 \\
\hline N1-C2-C3-C4 & 2 & -0.017 & -53.74 & -0.056 \\
\hline N1-C2-C3-C4 & 3 & -5.215 & -0.10 & -0.03 \\
\hline $\mathrm{C1}-\mathrm{C} 2-\mathrm{C} 3-\mathrm{C} 4$ & 4 & 0.027 & 0.18 & 0.00 \\
\hline $\mathrm{C} 1-\mathrm{C} 2-\mathrm{C} 3-\mathrm{C} 4$ & 5 & -0.009 & 0.03 & 0.02 \\
\hline \multirow[t]{3}{*}{$\mathrm{C1}-\mathrm{C} 2-\mathrm{C} 3-\mathrm{C} 4$} & 6 & -0.013 & 0.037 & 0.07 \\
\hline & & & transoid & \\
\hline & & $\mathrm{Xt}$ & $\mathrm{XtNp}$ & XtN'p \\
\hline N1-C2-C3-N4 & 1 & -0.047 & -0.09 & -0.08 \\
\hline N1-C2-C3-C4 & 2 & 0.009 & -0.04 & -0.02 \\
\hline $\mathrm{C1} 1-\mathrm{C} 2-\mathrm{C} 3-\mathrm{C} 4$ & 4 & 0.016 & 0.02 & 0.02 \\
\hline \multirow[t]{2}{*}{ HF } & & \multicolumn{3}{|c|}{ cisoid } \\
\hline & & Xs & $\mathrm{XsNp}$ & XsN'p \\
\hline N1-C2-C3-N4 & 1 & -43.50 & -0.008 & -0.055 \\
\hline N1-C2-C3-C4 & 2 & -27.96 & -14.34 & -41.03 \\
\hline N1-C2-C3-C4 & 3 & -15.14 & -44.20 & -13.10 \\
\hline C1-C2-C3-C4 & 4 & -40.21 & -40.98 & -42.51 \\
\hline $\mathrm{C1}-\mathrm{C} 2-\mathrm{C} 3-\mathrm{C} 4$ & 5 & -43.23 & -48.09 & -33.80 \\
\hline \multirow[t]{3}{*}{$\mathrm{C} 1-\mathrm{C} 2-\mathrm{C} 3-\mathrm{C} 4$} & 6 & -43.57 & -40.21 & -40.20 \\
\hline & & & transoid & \\
\hline & & $\mathrm{Xt}$ & $\mathrm{XtNp}$ & $\mathrm{XtN} p$ \\
\hline N1-C2-C3-N4 & 1 & -0.029 & 34.34 & -25.73 \\
\hline N1-C2-C3-C4 & 2 & -25.63 & -23.83 & -41.32 \\
\hline $\mathrm{C} 1-\mathrm{C} 2-\mathrm{C} 3-\mathrm{C} 4$ & 4 & -36.60 & -42.35 & -42.27 \\
\hline \multirow[t]{2}{*}{ B3LYP } & & \multicolumn{3}{|c|}{ cisoid } \\
\hline & & Xs & $\mathrm{XsNp}$ & XsN'p \\
\hline N1-C2-C3-N4 & 1 & -35.09 & -0.055 & -0.027 \\
\hline N1-C2-C3-C4 & 2 & -20.84 & -0.015 & -35.18 \\
\hline N1-C2-C3-C4 & 3 & -15.14 & -35.68 & -0.01 \\
\hline $\mathrm{C1}-\mathrm{C} 2-\mathrm{C} 3-\mathrm{C} 4$ & 4 & -35.60 & -34.19 & -35.04 \\
\hline
\end{tabular}


Table 8. Continued

\begin{tabular}{ccccc}
\hline & Molecule & \multicolumn{2}{c}{ Neutral } & First Protonation \\
\hline $\begin{array}{c}\text { Dihedral } \\
\text { Angels }\end{array}$ & & \multicolumn{3}{c}{ cisoid } \\
\hline B3LYP & & $\mathrm{Xs}$ & $\mathrm{XsNp}$ & XsN'p \\
C1-C2-C3-C4 & $\mathbf{5}$ & -43.23 & -48.09 & -33.80 \\
C1-C2-C3-C4 & $\mathbf{6}$ & -36.19 & -31.65 & -31.63 \\
& & & transoid & \\
& & $\mathrm{Xt}$ & $\mathrm{XtNp}$ & $\mathrm{XtN} p$ \\
N1-C2-C3-N4 & $\mathbf{1}$ & -0.025 & 13.46 & -13.40 \\
N1-C2-C3-C4 & $\mathbf{2}$ & -17.90 & -8.19 & -34.88 \\
C1-C2-C3-C4 & $\mathbf{4}$ & -26.11 & -34.20 & -34.35 \\
\hline
\end{tabular}

\section{Nucleophilicity Criteria}

The hard/soft base principle has been applied to kinetic phenomena for a long time. In this connection, organic chemistry has provided most of the examples, because the organic reactions are often slow enough for their rates to be easily measured. In organic chemistry, we are generally interested in the reactions of electrophiles and nucleophiles. These reactions are a particular kind of the general acid-with-base type of reaction, and so the principle of hard and soft acids and bases applies equally to the reactions of electrophiles and nucleophiles. So the acidity and basicity can be related to the theoretical interpretation of hard-soft acids and bases.

The solvated proton is a hard electrophile and little affected by frontier orbital interactions. For this reason, the $\mathrm{pK}_{\mathrm{a}}$ of the conjugated acid of a nucleophile is a good measure of the rate at which that nucleophile will attack to another hard electrophiles. ${ }^{48}$

Taking all those above mentioned points into account we have attempted to search a possible correlations between the experimental and computed acidity constants, $\mathrm{pK}_{\mathrm{a}}$ values, and computed nucleophilicity of the studied molecules. The unusual behaviour of any molecule which will cause a deviation form correlation line then would let us to think the external effects such as solvation on this molecule in such a way to alter the nucleophilicity-electrophilicity or/and acidity-basicity character of the molecule at a great extend.

The nucleophilicity, $\mathrm{n}=\mathrm{E}_{\mathrm{HOMO}}-\mathrm{E}_{\mathrm{LUMO}}{ }^{49}$ of studied molecules were calculated and the aqueous phase calculated $\mathrm{n}$ values of the studied neutral molecules were depicted in Table 9 .

The best fit of nucleophilicity to $\mathrm{pK}_{\mathrm{a}}$ values comes from PM5 calculated nucleophilicities and PM5 calculated $\mathrm{pK}_{\mathrm{a}}$ values (Figure 2). 


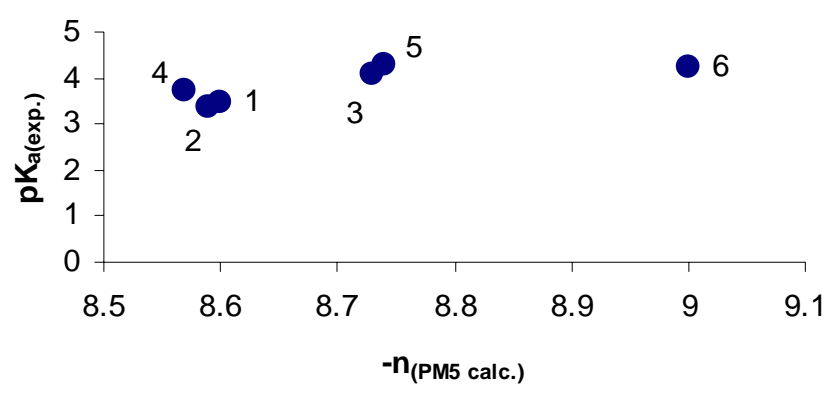

Figure 2. The plot of PM5 aqueous phase calculated nucleophilicities, -n values, and experimental acidity constants, $\mathrm{pK}_{\mathrm{a} \text { (exp.), }}$, for studied bipyridine derivatives.

Table 9. Aqueous phase PM5, HF and B3LYP calculated nucleophilicty values for neutral molecules

\begin{tabular}{cccc}
\hline Molecule & $E_{\text {HOMO }}$ & $E_{L U M O}$ & $\mathrm{n}^{\mathrm{a}}$ \\
\hline $\mathbf{P M 5}$ & & & \\
$\mathbf{1 s}$ & -9.443 & -0.823 & -8.620 \\
$\mathbf{1 t}$ & -9.405 & -0.800 & -8.605 \\
$\mathbf{2 s}$ & -9.355 & -0.760 & -8.595 \\
$\mathbf{2 t}$ & -9.363 & -0.756 & -8.607 \\
$\mathbf{3}$ & -9.628 & -0.890 & -8.738 \\
$\mathbf{4 s}$ & -9.293 & -0.721 & -8.572 \\
$\mathbf{4 t}$ & -9.291 & -0.721 & -8.570 \\
$\mathbf{5}$ & -9.591 & -0.851 & -8.740 \\
$\mathbf{6}$ & -9.985 & -0.979 & -9.006 \\
$\mathbf{H F}$ & & & \\
& & & \\
$\mathbf{1 s}$ & -0.32 & 0.09 & -0.41 \\
$\mathbf{1 t}$ & -0.31 & 0.08 & -0.39 \\
$\mathbf{2 s}$ & -0.31 & 0.08 & -0.39 \\
$\mathbf{2 t}$ & -0.31 & 0.08 & -0.39 \\
$\mathbf{3}$ & -0.32 & 0.07 & -0.39 \\
$\mathbf{4 s}$ & -0.30 & 0.08 & -0.38 \\
$\mathbf{4 t}$ & -0.30 & 0.09 & -0.39 \\
$\mathbf{5}$ & -0.33 & 0.08 & -0.41 \\
$\mathbf{6}$ & -0.34 & 0.08 & -0.42 \\
$\mathbf{B 3 L Y P}$ & & & \\
$\mathbf{1 s}$ & -0.24 & -0.04 & -0.20 \\
\hline & & &
\end{tabular}


Table 9. Continued

\begin{tabular}{cccc}
\hline Molecule & EHOMO & ELUMO & na \\
\hline $\mathbf{1 t}$ & -0.23 & -0.05 & -0.18 \\
$\mathbf{2 s}$ & -0.23 & -0.05 & -0.18 \\
$\mathbf{2 t}$ & -0.23 & -0.05 & -0.18 \\
$\mathbf{3}$ & -0.24 & -0.05 & -0.19 \\
$\mathbf{4 s}$ & -0.23 & -0.04 & -0.19 \\
$\mathbf{4 t}$ & -0.23 & -0.04 & -0.19 \\
$\mathbf{5}$ & -0.25 & -0.04 & -0.21 \\
$\mathbf{6}$ & -0.26 & -0.05 & -0.21 \\
\hline
\end{tabular}

${ }^{a} \mathrm{n}=\mathrm{E}_{\text {HOMO }}-\mathrm{E}_{\text {LUMO }}$

The lower solvation effect on compounds 1, 2 and $\mathbf{4}$ comparing to compounds $\mathbf{3}$ and $\mathbf{5}$ is reflected by decrease in nucleophilicities. It seems that the most solvated molecule among the others is compound $\mathbf{6}$ which possess two nitrogen atoms which are positioned very suitably for solvation after protonation and form paraquat like stable dication which plays a very important role in electron transport phenomena. ${ }^{50}$

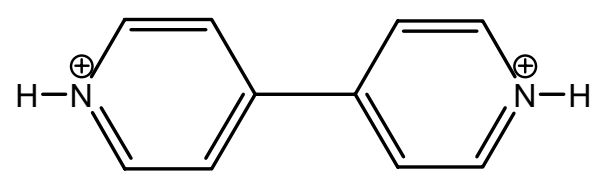

It seems that the nucleophilicity is more substituent dependant than that of ionization constant, $\mathrm{K}_{\mathrm{a}}$ value, for these derivatives. This means that the position of substituent is also an important factor and effects the electronic changes on the protonation center.

\section{Acknowledgement}

Our research group greatly in dept to Eskişehir Osmangazi University for providing the Gaussian 03 and MOPAC 2002 program through Research Project number 200519010.

\section{References}

1. Kawai, T.; Furukawa, N.; Oae, S. Tetrahedron Lett. 1984, 25, 2549.

2. Shibutani, T.; Fujihawa, H.; Furukawa, N.; Oae, S. Heteroatom Chem. 1991, 2, 521.

3. Furukawa, N.; Ogawa, S.; Matsumura, K. M.; Fujihara, H. J. Org. Chem. 1991, 56, 6341. 
4. Uchida, Y.; Onoue, K.; Tada, N.; Nagao F.; Oae S. Tetrahedron Lett. 1989, 30, 567.

5. Uchida, Y.; Kozawa, H.; Oae, S. Tetrahedron Lett. 1989, 30, 6365.

6. Uchida, Y.; Takaya, Y.; Oae, S. Heterocycles 1990, 30, 347.

7. Uchida, Y.; Onoue, K.; Tada, N.; Nagao F.; Kosawa, H.; Oae, S. Heteroatom Chem. 1990, 1, 295.

8. Uchida, Y.; Kawai, M.; Masauji, H.; Oas, S. Heteroatom Chem. 1993, 4, 421.

9. Uchida, Y.; Kajita, R.; Kawasaki, Y.; Oae, S. Tetrahedron Lett. 1995, 36, 4077.

10. Chisholm, M. H.; Huffman, J. C.; Rothwell, I. P.; Bradley, P. G.; Kress, N.; Woodruff, W. H. J. Am. Chem. Soc. 1981, 103, 4945.

11. Roodt, A.; Leipoldt, J. G.; Deutsch, E. A.; Sullivan, J. C. Inorg. Chem. 1992, 31,1080.

12. Benedix, R.; Birner, P.; Hennig, H. J. Mol. Struct. Theochem 1982, 90, 65.

13. Galasso, V.; De Alti, G.; Bigotto, A. Tetrahedron 1971, 27, 991.

14. Szabo, K.; Kunsagi-Mate, S.; Marek, N. J. Mol. Struct. Theochem 1995, 333, 275.

15. Hofmann, H. J. J.Mol. Struct. Theochem 1986, 139, 213.

16. Lunn, G. J. Org. Chem. 1992, 57, 6317.

17. Balzani, V.; Bolletta, F.; Gandolfi, M. T.; Maestri, M.; Top, M. Curr. Chem. 1978, 75, 1.

18. Kalyanasundaram, K. Photochemistry of Polypyridine and Porpyrin Complexes, Academic Press, London, 1992.

19. Grummt, U. V.; Erhardt S. J. Mol. Struct. Theochem 2004, 685, 133.

20. Göller, A.; Grummt, U. W.; Chem. Phys. Lett. 2000, 321, 399.

21. Göller, A.; Grummt, U. W.; Chem. Phys. Lett. 2002, 354, 233.

22. Creaven, B. S.; Long, C.; Howie, R. A.; McQuillan, G. P.; Low, J. Inorg. Chim. Acta 1989, 157, 151.

23. Uson, R.; Forniers, J.; Tomas, M.; Casas, J. M.; Fortuno C. Polyhedron 1989, 8, 2209.

24. Onggo, D.; Craig, D. C.; Rae A. D.; Goodwin H. A. Aust. J. Chem. 1991, 44,219.

25. Fornies, J.; Navarro, R.; Sicilia V.; Tomas M. Inorg. Chem. 1993, 32, 3675.

26. Koning, B.; de Boer, J.H.; Meetsma A. and Kellogg R.M. Arkivoc 2004, (ii), 189

27. Dietrich-Buchecker, C. O.; Sauvage J.-P. Chem. Rev., 1987, 87, 795.

28. Lehn J.-M. Angew. Chem., Int. Ed. Eng. 1990, 29, 1304.

29. Thummel, R. P. Tetrahedron 1991, 47, 6851.

30. Constable, E. C. Tetrahedron 1992, 48, 10013.

31. Potts, K. T.; Gheysenraiford, K. A.; Keshavarz, K. M. J. Am. Chem. Soc. 1993, 115, 2793.

32. Constable, E. C. Prog. Inorg. Chem. 1994, 42, 67.

33. Amabilino, D. B.; Stoddart, J. F. Chem. Rev. 1995, 95, 2725.

34. Kurkcuoglu, G. S.; Kavlak, I.; Arslan, T.; Ogretir, C. J. Mol. Model. 2009, 15, 79.

35. Öğretir, C.; Tay, N. F.; Oztürk, I, I. J. Mol. Graph. Model. 2007, $26,740$.

36. Tokay, N.; Ogretir, C. J.Mol. Struct. Theochem 2003, 626,113.

37. Civcir, P.U.; Ogretir, C. J. Mol. Struct. Theochem 2000, 507, 39.

38. Öğretir, C.; Bereket, G.; Poyraz, M. Turkish VIII th National Symposium on Chemistry and Chemical Engineering 1992, 518, 153. 
39. Öğretir, C.; Berber, H.; Kanışkan, N. Turkish VIII th National Symposium on Chemistry and Chemical Engineering 1992, 518, 163.

40. Öğretir, C., Tokay, N. J. Mol. Struct. Theochem 2004, 679, 1.

41. (a) Stewart, J. J. P. MOPAC 2002 implemented in Cache Work System Pro., Version 6.1, Fujitsu Ltd. (b) Frish, M. J.; Trucks, G. W.; Schlegel, H. B.; Gill, P. M. W.; Johnson, B. G.; Wong, M. W.; Foresman, J.B.; Robb M. A.; HeadGordon M.; Replogle, E.S.; Gomperts, R.; Martin, J. L.; Fox, D. Y.; Defress, D. J.; Baker, J.; Stewart, J. J. P.; Pople, J.A., GAUSSIAN 03, Gaussian Inc., Pittsburg PA, 2003.

42. CS ChemOffice Pro for Microsoft Windows, Cambridge Scientific Computing Inc., 875 Massachusetts Avenue, Suite \&1, Cambridge MA, 02139, USA.

43. Öğretir, C.; Özöğüt, D.;Yarlıgan, S.; Arslan, T. J. Mol. Struct. Theochem 2006, 759, 73

44. Sefc1k, J.; Goddard III, W. A. Geochimica et Cosmochimica Acta 2001, 65, 4435.

45. Speranza, M. Adv. Heterocycl. Chem. 1985, 40, 25.

46. Albert, A. Physical Methods in Heterocyclic Chemistry. Katritzky, A. R. (Ed.) Academic Press: New York, 1963; Vol.1, pp 61-67.

47. Johnson, C. D. The Hammett Equation, Cambridge University Press, London, 1973.

48. Fleming, I. Frontier Orbitals and Organic Chemical Reactions John W1ley \& Sons, 1982.

49. Hemmateenejad, B.; Safarpour, M. A.; Taghavi, F. J. Mol. Struct. Theochem 2003, 635, 183.

50. Katritzky, A. R.; Pozharskii, A. F.; Soldatenkov, A. T. Heterocycles in life and society, Wiley, 1997; p 168 